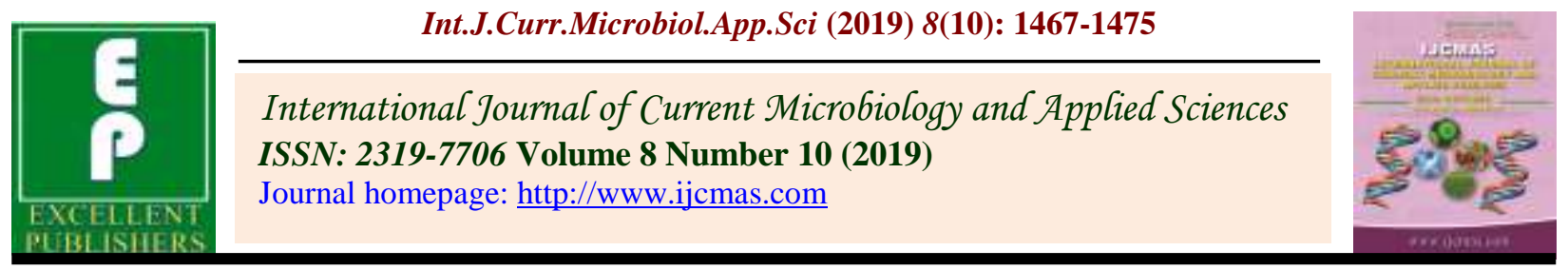

Original Research Article

https://doi.org/10.20546/ijcmas.2019.810.172

\title{
Efficacy of Traditional Seed Dressers and Oils on Isabgol Seeds against Alternaria alternata and Fusarium semitectum under Storage Conditions
}

\author{
M. L. Meena ${ }^{1}$, R. P. Maharshi ${ }^{2}$ and S. K. Bairwa ${ }^{3}$ \\ ${ }^{1}$ Department of Plant Pathology, SKN College of Agriculture, (SK Rajasthan Agricultural \\ University, Bikaner), Jobner-303329, Jaipur, Rajasthan, India \\ ${ }^{2}$ Department of Hort, College of Agriculture, Lalsot (Dausa)-303 511, India \\ ${ }^{3}$ Department of Plant Pathology, College of Agriculture, Lalsot (Dausa), India
}

*Corresponding author

Keywords

Isabgol, Alternaria alternata, Fusarium semitectum, Seed dresser, Oil, Storage

Article Info

Accepted:

12 September 2019

Available Online:

10 October 2019
In seeds of Isabgol, test of relative efficacy of four traditional seed dresser like tulsi leaf powder, mehandi leaves, turmeric powder and neem leaf powder revealed that neem leaf powder gave effective control of Alternaria alternate and Fusarium semitectum are followed by tulsi leaf powder and which resulted increased into germination even after 6 month storage as compared to control. Four oils like neem, mustard, castorl and palas oil were used for seed treatment and it was observed that the neem oil gave effective control even after six months of storage as compared to control.

\section{Introduction}

Isabgol (Plantago ovate Forsk.) is one of the most important medicinal crops in India. It is mainly grown in Gujarat, Rajasthan and Madhya Pradesh. However, the crop is spreading to other non-traditional parts of the country such as Haryana, Uttar Pradesh and Karnataka. In Rajasthan, it is being cultivated in 4,17,109 hectare area with a total production of 3,41,447 tonnes of seeds with an average productivity $891 \mathrm{~kg} \mathrm{ha}^{-1}$ (Anonymous, 2017-18). It is mainly grown for husk purpose, which is used for medicinal purpose for controlling constipations, irritation, diarrhoea, chronic dysenteries of amoebic and bacilliary origin and ulcerated surface of intestinal mucosa (Choudhary, 2015). Alternaria leaf spot of isabgol caused by Alternaria alternata, has become a severe problem in Isabgol growing areas of Rajasthan (Bajaya et al., 2017). Although, only a few reports are available on plant drug (Chourasia, 1995).

But presence of mycotoxins in Agricultural commodities has been well investigated from different parts of India and abroad (Sinha and Kumari, 1990 and Bajaya et al., 2017). 
Detection of fungal inoculums on seed lot is important in order to avoid / reduce the risk during standing crop as well as under storage conditions. Elwakil and Ghoneem (1999) reported 41 species of fungi belonging to 21 genera from commercial seed samples of Isabgol from Egypt.

\section{Materials and Methods}

\section{Management of seed borne fungi}

Incidence of seed borne fungi and disease caused was managed under storage conditions.

\section{Under storage condition}

Seeds of sample IG -3 which revealed higher incidence of pathogenic fungi were artificially inoculated with 10 days old culture of test fungus (Alternaria alternata and Fusarium semitectum) and then separately treated with traditional seed treating materials and oils (edible and non-edible). A sample of $500 \mathrm{~g}$ seed was treated with each of the following at the mentioned dose.

\section{Traditional seed treating material}

Traditional seed treating materials mentioned below were applied to the seeds as dry powder @ $20.0 \mathrm{~g} \mathrm{~kg}^{-1}$ seed. Proper control was also maintained

Tulsi leaves powder (Ocimum basilicum L.)

Mehndi leaf powder (Lawsonia inermis L.)

Turmeric powder (Curcuma longa L.)

Neem leaf powder (Azadirachta indica A. Juss)

Control (Untreated)
The seeds were shaken with traditional seed materials and oils on a mechanical rotary shaker for 2 hours to give a uniform coating. The seeds were then kept in polythene bags and stored at $28+2^{\circ} \mathrm{C}$. The seeds were tested for incidence of Alternaria alternata and Fusarium semitectum and seed germination 0 , 2, 4 and 6 months of storage by Blotter paper Method and Rolled Paper Towel Method, respectively.

\section{Oils}

Following oils were used @ $10 \mathrm{ml} \mathrm{kg}^{-1}$ seeds.

Neem (Azadirachta indica A. Juss)

Mustard (Brassica campestris var. sarson Prain)

Castor (Ricinus communis L.)

Palas (Butea frondosa Konig)

Control (Treated)

Control (Untreated)

In case of non-edible oils, equal amount of acetone was added to reduce the viscosity of oil for proper coating of the seed. Proper control was maintained.

\section{Results and Discussion}

Effect of traditional seed treating material on incidence of Alternaria alternata and per cent germination of seeds

Among seeds treated with different traditional seed treating material, the minimum incidence of Alternaria alternata was observed in neem leaf powder $(10.76 \%)$ followed by tulsi leaf powder (12.23\%) after 2, 4 and 6 months of storage. Germination was also higher in seed 
treated with these materials. Reduction in $A$. alternata and increase in germination were significantly higher in all the seed treating materials at all level of storage as compared to inoculated control.

With regard to interaction effect of six traditional seed treating material and 4 storage periods, minimum incidence of $A$. alternata was observed in neem leaf treated seeds at 0 month of storage followed by tulsi leaf powder at 0 month and neem leaf powder at 6 months. Maximum germination was also recorded in these treatments at the same storage period. These treatment were highly significant to each other as compared to control (Table 1).

Effect of traditional seed treating material on incidence of $F$. semitectum and germination of seeds

Among seeds treated with different traditional seed treating materials, minimum incidence of $F$. semitectum was observed in neem leaf powder treated seeds $(12.23 \%)$ followed by tulsi leaf power (12.92\%) after 2, 4 and 6 months of storage. The germination was also higher in seed treated with these treatments.

Reduction in incidence of $F$. semitectum and increase in germination were significant in all the seed treating materials at all level of storage as compared to inoculated untreated control.

Interaction effect between six traditional seed treating materials and four storage periods revealed minimum incidence of $F$. semitectum in neem leaf treated seed at 0 month followed by tulsi leaf powder treated at 0 month. Neem leaf powder treated seed also showed maximum seed germination at the same storage period. These treatments were highly significant to each other as compared to control (Table 2).

\section{Effect of oils on incidence of Alternaria alternata and germination of seeds}

Among seeds treated with different oils just after harvest, minimum incidence of $A$. alternata was observed with neem oil (9.97\%) followed by mustard oil (12.23\%). Germination was also highest in seed treatment with neem oil $(68.90 \%)$. After 2, 4 and 6 months of storage, minimum in incidence of A. alternata were recorded in seed treated with neem oil followed by mustard oil. Reduction in incidence of $A$. alternata and increase in germination per cent were significant in all the treatment at all levels of storage as compared to inoculated control.

With regard interaction effect between six oils and four storage periods, it was observed that minimum incidence of A. alternata occurred in neem oil treated seeds $(5.74 \%)$ at 6 months followed by neem oil at 4 months $(8.13 \%)$. Maximum seed germination was recorded in neem oil at six month followed by neem oil at 4 month and mustard oil at 6 month respectively. These treatments were highly significant to each other as compared to control (Table 3).

\section{Effect of oils on incidence of $\boldsymbol{F}$. semitectum and germination of seeds}

Among seeds treated with different oils just after harvest minimum incidence of $F$. semitectum was observed with neem oil (12.92\%) followed by mustard oil (14.76\%).

Germination was also highest in seed treated with neem oil $(68.15 \%)$ after 2,4 and 6 months of storage. The germination was also higher in seed treated with these oils.

Reduction in incidence of $F$. semitectum and increase in germination were significant in all the treatments at all levels of storage period as compared to inoculated control. 
Table.1 Effect of different traditional seed treating materials and storage period on incidence of Alternaria alternata and germination of Isabgol seeds

\begin{tabular}{|c|c|c|c|c|c|c|c|c|c|c|c|c|c|c|c|c|c|}
\hline \multirow{4}{*}{$\begin{array}{c}\text { Traditiona } \\
\text { I treating } \\
\text { materials } \\
\begin{array}{c}\text { Tulsi leaf } \\
\text { powder }\end{array}\end{array}$} & \multirow{4}{*}{$\begin{array}{c}\text { Dose } \\
(\mathbf{g} \\
\mathbf{k g}^{-1} \\
\text { seed } \\
) \\
20.0\end{array}$} & \multicolumn{16}{|c|}{ Storage period ( Months) } \\
\hline & & \multicolumn{4}{|c|}{$\mathbf{0}$} & \multicolumn{4}{|c|}{2} & \multicolumn{4}{|c|}{4} & \multicolumn{4}{|c|}{6} \\
\hline & & \multicolumn{2}{|c|}{$\begin{array}{c}\text { Incidence } \\
(\%)\end{array}$} & \multicolumn{2}{|c|}{$\begin{array}{c}\text { Germinatio } \\
\text { n }(\%)\end{array}$} & \multicolumn{2}{|c|}{$\begin{array}{c}\text { Incidence } \\
(\%)\end{array}$} & \multicolumn{2}{|c|}{$\begin{array}{c}\text { Germinatio } \\
\text { n }(\%)\end{array}$} & \multicolumn{2}{|c|}{$\begin{array}{c}\text { Incidence } \\
(\%)\end{array}$} & \multicolumn{2}{|c|}{$\begin{array}{c}\text { Germinatio } \\
\text { n }(\%)\end{array}$} & \multicolumn{2}{|c|}{$\begin{array}{c}\text { Incidence } \\
(\%)\end{array}$} & \multicolumn{2}{|c|}{$\begin{array}{c}\text { Germinatio } \\
\mathbf{n}(\%)\end{array}$} \\
\hline & & 4.5 & $\begin{array}{c}(12.23 \\
)\end{array}$ & 82.0 & $(64.98)$ & 5.5 & $\begin{array}{c}(13.55 \\
)\end{array}$ & 80.0 & $(63.45)$ & 5.0 & $\begin{array}{c}(12.92 \\
)\end{array}$ & $\begin{array}{c}82.0 \\
0\end{array}$ & $(64.92)$ & $\begin{array}{l}4 . \\
0\end{array}$ & $\begin{array}{c}(11.54 \\
)\end{array}$ & 84.0 & $(66.52)$ \\
\hline $\begin{array}{c}\text { Mehandi } \\
\text { leaf powder }\end{array}$ & 20.0 & 6.0 & $\begin{array}{c}(14.16 \\
)\end{array}$ & 77.0 & $(61.35)$ & 7.0 & $\begin{array}{c}(15.32 \\
)\end{array}$ & 76.0 & $(60.79)$ & 5.0 & $\begin{array}{c}(12.92 \\
)\end{array}$ & $\begin{array}{c}82.0 \\
0\end{array}$ & $(65.11)$ & $\begin{array}{l}4 . \\
5\end{array}$ & $\begin{array}{c}(12.23 \\
)\end{array}$ & 82.0 & $(64.93)$ \\
\hline $\begin{array}{c}\text { Turmeric } \\
\text { powder }\end{array}$ & 20.0 & 7.0 & $\begin{array}{c}(15.34 \\
)\end{array}$ & 76.0 & (60.68) & 7.5 & $\begin{array}{c}(15.89 \\
)\end{array}$ & 76.0 & $(60.70)$ & 6.0 & $\begin{array}{c}(14.18 \\
)\end{array}$ & $\begin{array}{c}77.2 \\
5\end{array}$ & (61.56) & $\begin{array}{l}5 . \\
0\end{array}$ & $\begin{array}{c}(12.92 \\
)\end{array}$ & 82.0 & $(64.97)$ \\
\hline $\begin{array}{c}\text { Neem leaf } \\
\text { powder }\end{array}$ & 20.0 & 3.5 & $\begin{array}{c}(10.76 \\
)\end{array}$ & 87.0 & (68.96) & 4.0 & $\begin{array}{c}(11.54 \\
)\end{array}$ & 84.0 & $(66.52)$ & 3.5 & $\begin{array}{c}(10.76 \\
)\end{array}$ & $\begin{array}{c}87.0 \\
0\end{array}$ & (68.96) & $\begin{array}{l}3 . \\
5\end{array}$ & $\begin{array}{c}(10.76 \\
)\end{array}$ & 87.0 & $(69.20)$ \\
\hline Inoculated & & $\begin{array}{c}10 . \\
0\end{array}$ & $\begin{array}{c}(18.43 \\
)\end{array}$ & 72.0 & (58.06) & $\begin{array}{c}12 . \\
0\end{array}$ & $\begin{array}{c}(20.27 \\
)\end{array}$ & 69.0 & (56.17) & $\begin{array}{c}12 . \\
0\end{array}$ & $\begin{array}{c}(20.27 \\
)\end{array}$ & $\begin{array}{c}71.0 \\
0\end{array}$ & (57.43) & $\begin{array}{l}9 . \\
0\end{array}$ & $\begin{array}{c}(17.46 \\
)\end{array}$ & 75.0 & $(60.03)$ \\
\hline $\begin{array}{c}\text { Un } \\
\text { Inoculated }\end{array}$ & & 7.0 & $\begin{array}{c}(15.34 \\
)\end{array}$ & 78.0 & (62.04) & 9.0 & $\begin{array}{c}(17.46 \\
)\end{array}$ & 74.5 & (59.68) & 6.0 & $\begin{array}{c}(14.13 \\
)\end{array}$ & $\begin{array}{c}79.0 \\
0\end{array}$ & (62.74) & $\begin{array}{l}5 . \\
5\end{array}$ & $\begin{array}{c}(13.55 \\
)\end{array}$ & 77.5 & (61.75) \\
\hline SEm \pm & & & 0.32 & & 0.91 & & 0.27 & & 1.15 & & 0.34 & & 1.23 & & 0.29 & & 1.46 \\
\hline CD at $5 \%$ & & & 0.95 & & 2.70 & & 0.81 & & 3.41 & & 1.01 & & 3.64 & & 0.85 & & 4.35 \\
\hline CV (\%) & & & 4.43 & & 3.75 & & 3.46 & & 3.75 & & 4.78 & & 3.86 & & 4.39 & & 4.53 \\
\hline
\end{tabular}

\begin{tabular}{|c|c|c|c|c|c|c|c|}
\hline \multicolumn{4}{|c|}{ Interaction of treatment and month for per cent incidence } & \multicolumn{4}{|c|}{ Interaction of treatment and month for per cent germination } \\
\hline SOV & SEm \pm & $\mathrm{CD}$ at $5 \%$ & $\mathrm{CV}(\%)$ & SOV & $\mathrm{SEm} \pm$ & $\begin{array}{c}\mathrm{CD} \text { at } 5 \\
\%\end{array}$ & $\mathrm{CV}(\%)$ \\
\hline TRAD & 0.153 & 0.430 & 4.260 & TRAD & 0.60 & 1.70 & 3.82 \\
\hline $\mathbf{M}$ & 0.115 & 0.325 & & M & 0.45 & 1.28 & \\
\hline TRADXM & 0.305 & 0.861 & & TRADXM & 1.20 & 3.39 & \\
\hline
\end{tabular}


Table.2 Effect of different traditional seed treating materials and storage period on incidence of Fusarium semitectum and germination of Isabgol seeds

\begin{tabular}{|c|c|c|c|c|c|c|c|c|c|c|c|c|c|c|c|c|c|}
\hline \multirow{4}{*}{$\begin{array}{c}\text { Traditional } \\
\text { treating } \\
\text { materials } \\
\begin{array}{c}\text { Tulsi leaf } \\
\text { powder }\end{array}\end{array}$} & \multirow{4}{*}{$\begin{array}{c}\begin{array}{c}\text { Dose } \\
\left(\mathrm{g} \mathrm{kg}^{-1}\right. \\
\text { seed })\end{array} \\
20.0\end{array}$} & \multicolumn{16}{|c|}{ Storage period ( Months) } \\
\hline & & \multicolumn{4}{|c|}{$\mathbf{0}$} & \multicolumn{4}{|c|}{2} & \multicolumn{4}{|c|}{4} & \multicolumn{4}{|c|}{6} \\
\hline & & \multicolumn{2}{|c|}{$\begin{array}{c}\text { Incidence } \\
(\%)\end{array}$} & \multicolumn{2}{|c|}{$\begin{array}{c}\text { Germination } \\
(\%)\end{array}$} & \multicolumn{2}{|c|}{$\begin{array}{c}\text { Incidence } \\
(\%)\end{array}$} & \multicolumn{2}{|c|}{$\begin{array}{c}\text { Germination } \\
(\%)\end{array}$} & \multicolumn{2}{|c|}{$\begin{array}{c}\text { Incidence } \\
(\%)\end{array}$} & \multicolumn{2}{|c|}{$\begin{array}{l}\text { Jermination } \\
(\%)\end{array}$} & \multicolumn{2}{|c|}{$\begin{array}{c}\text { Incidence } \\
(\%)\end{array}$} & \multicolumn{2}{|c|}{$\begin{array}{c}\text { Germination } \\
(\%)\end{array}$} \\
\hline & & 5.0 & $(12.92)$ & 82.0 & $(64.92)$ & 6.00 & (14.18) & 76.0 & $(60.68)$ & 6.50 & $(14.76)$ & 80.0 & $(63.46)$ & 5.5 & $(13.55)$ & 83.0 & $(65.71)$ \\
\hline $\begin{array}{c}\text { Mehandi } \\
\text { leaf powder }\end{array}$ & 20.0 & 7.0 & $(15.34)$ & 78.0 & $(62.06)$ & 7.75 & (16.16) & 75.0 & $(60.02)$ & 7.50 & (15.89) & 79.0 & (62.79) & 6.5 & (14.76) & 79.0 & (62.74) \\
\hline $\begin{array}{l}\text { Turmeric } \\
\text { powder }\end{array}$ & 20.0 & 8.0 & $(16.40)$ & 75.0 & $(60.02)$ & 7.75 & (16.16) & 72.0 & $(58.07)$ & 7.50 & (15.89) & 78.0 & (62.06) & 7.0 & $(15.32)$ & 78.0 & 62.04) \\
\hline $\begin{array}{l}\text { Neem leaf } \\
\text { powder }\end{array}$ & 20.0 & 4.5 & $(12.23)$ & 85.0 & (67.36) & 5.50 & (13.55) & 83.0 & $(65.71)$ & 6.00 & (14.18) & 81.0 & (64.18) & 5.0 & (12.92) & 85.0 & (67.27) \\
\hline Inoculated & 20.0 & 10.0 & $(18.43)$ & 72.0 & (58.06) & 12.00 & $(20.23)$ & 69.0 & $(56.17)$ & 11.75 & (20.04) & 71.0 & (57.43) & 9.0 & (17.46) & 75.0 & $(60.03)$ \\
\hline $\begin{array}{c}\text { Un } \\
\text { Inoculated }\end{array}$ & 20.0 & 7.0 & (15.34) & 78.0 & (62.04) & 9.00 & (17.46) & 74.5 & $(59.68)$ & 6.00 & (14.13) & 79.0 & (62.74) & 5.5 & (13.55) & 77.5 & (61.75) \\
\hline SEm \pm & & & 0.30 & & 0.96 & & 0.39 & & 0.79 & & 0.38 & & 0.86 & & 0.31 & & 0.99 \\
\hline CD at $5 \%$ & & & 0.89 & & 2.85 & & 1.17 & & 2.35 & & 1.12 & & 2.55 & & 0.93 & & 2.94 \\
\hline CV (\%) & & & 3.94 & & 2.64 & & 4.84 & & 2.64 & & 4.76 & & 2.77 & & 4.29 & & 3.13 \\
\hline
\end{tabular}

\begin{tabular}{|c|c|c|c|c|c|c|c|}
\hline \multicolumn{2}{|c|}{ Interaction of treatment and month for per cent incidence } & \multicolumn{3}{|c|}{ Interaction of treatment and month for per cent germination } \\
\hline SOV & SEm \pm & CD at $5 \%$ & CV $(\%)$ & SOV & SEm \pm & CD at 5 \\
$\%$ & & & & \\
CV $(\%)$ \\
\hline TRAD & 0.174 & 0.490 & 4.500 & TRAD & 0.45 & 1.27 \\
\hline M & 0.131 & 0.370 & & M & 0.34 & 0.96 \\
\hline TRADXM & $\mathbf{0 . 3 4 8}$ & $\mathbf{0 . 9 8 0}$ & & TRADXM & $\mathbf{0 . 9 0}$ & $\mathbf{2 . 5 5}$ \\
\hline
\end{tabular}


Table.3 Effect of different oils and storage period on incidence of Alternaria alternata and germination of Isabgol seeds

\begin{tabular}{|c|c|c|c|c|c|c|c|c|c|c|c|c|c|c|c|c|c|}
\hline \multirow[t]{3}{*}{ Oils } & \multirow{3}{*}{$\begin{array}{c}\text { Dose } \\
(\mathrm{ml} \\
\mathbf{k g}^{-1} \\
\text { seed) }\end{array}$} & \multicolumn{16}{|c|}{ Storage period ( Months) } \\
\hline & & \multicolumn{4}{|c|}{0 Month } & \multicolumn{4}{|c|}{2 Month } & \multicolumn{4}{|c|}{4 Month } & \multicolumn{4}{|c|}{6 Month } \\
\hline & & \multicolumn{2}{|c|}{$\begin{array}{c}\text { Incidence } \\
(\%)\end{array}$} & \multicolumn{2}{|c|}{$\begin{array}{c}\text { Germination } \\
(\%)\end{array}$} & \multicolumn{2}{|c|}{$\begin{array}{c}\text { Incidence } \\
(\%)\end{array}$} & \multicolumn{2}{|c|}{$\begin{array}{c}\text { Germination } \\
(\%)\end{array}$} & \multicolumn{2}{|c|}{$\begin{array}{c}\text { Incidence } \\
(\%)\end{array}$} & \multicolumn{2}{|c|}{$\begin{array}{c}\text { Germination } \\
(\%)\end{array}$} & \multicolumn{2}{|c|}{$\begin{array}{c}\text { Incidence } \\
(\%)\end{array}$} & \multicolumn{2}{|c|}{$\begin{array}{c}\text { Germination } \\
(\%)\end{array}$} \\
\hline Neem oil & 10.0 & 3.0 & $(9.97)$ & 87.0 & $(68.90)$ & 3.5 & $(10.76)$ & 88.0 & $(69.82)$ & 2.0 & $(8.13)$ & 88.0 & $(69.84)$ & 1.0 & $(5.74)$ & 89.0 & (70.75) \\
\hline $\begin{array}{c}\text { Mustard } \\
\text { oil }\end{array}$ & 10.0 & 4.5 & (12.23) & 85.0 & $(67.29)$ & 5.0 & (12.92) & 82.0 & $(64.95)$ & 3.5 & $(10.76)$ & 84.0 & $(66.45)$ & 2.0 & $(8.13)$ & 86.0 & $(68.06)$ \\
\hline Castor oil & 10.0 & 6.5 & $(14.76)$ & 80.0 & $(63.48)$ & 6.5 & (14.76) & 78.0 & $(62.06)$ & 4.5 & $(12.23)$ & 83.0 & $(65.69)$ & 2.5 & $(9.05)$ & 85.0 & $(67.29)$ \\
\hline Palas oil & 10.0 & 6.5 & $(14.76)$ & 79.0 & $(62.77)$ & 6.5 & (14.76) & 79.0 & $(62.77)$ & 4.0 & (11.54) & 84.0 & $(66.45)$ & 3.0 & $(9.97)$ & 86.0 & (68.11) \\
\hline Inoculated & 10.0 & 10. & (18.43) & 72.0 & $(58.06)$ & 12.0 & (20.27) & 74.0 & $(59.41)$ & 11.5 & $(19.82)$ & 71.0 & $(57.43)$ & 9.0 & $(17.46)$ & 75.0 & $(60.03)$ \\
\hline $\begin{array}{c}\text { Un } \\
\text { Inoculated }\end{array}$ & & 7.0 & $(15.32)$ & 78.0 & $(62.04)$ & 9.0 & (17.46) & 74.5 & $(59.68)$ & 6.0 & $(14.18)$ & 79.0 & $(62.74)$ & 5.5 & $(13.55)$ & 77.5 & (61.75) \\
\hline SEm \pm & & & 0.32 & & 0.91 & & 0.27 & & 1.10 & & 0.27 & & 0.86 & & 0.26 & & 1.16 \\
\hline CD at $5 \%$ & & & 0.94 & & 2.70 & & 0.79 & & 3.27 & & 0.80 & & 2.56 & & 0.78 & & 3.44 \\
\hline CV (\%) & & & 4.43 & & 3.49 & & 3.53 & & 3.49 & & 4.19 & & 2.66 & & 4.94 & & 3.51 \\
\hline
\end{tabular}

\begin{tabular}{|c|c|c|c|c|c|c|c|}
\hline \multicolumn{4}{|c|}{ Interaction of treatment and month for per cent incidence } & \multicolumn{3}{|c|}{ Interaction of treatment and month for per cent germination } \\
\hline SOV & SEm \pm & CD at 5 \% & CV $(\%)$ & SOV & SEm \pm & CD at 5\% & CV (\%) \\
\hline O & 0.140 & 0.394 & 4.228 & O & 0.51 & 1.43 \\
\hline M & 0.106 & 0.297 & & M & 0.38 & 1.08 & 3.15 \\
\hline OXM & $\mathbf{0 . 2 7 9}$ & $\mathbf{0 . 7 8 7}$ & & OXM & $\mathbf{1 . 0 1}$ & $\mathbf{2 . 8 6}$ \\
\hline
\end{tabular}


Table.4 Effect of different oils and storage period on incidence of Fusarium semitectum and germination of Isabgol seeds

\begin{tabular}{|c|c|c|c|c|c|c|c|c|c|c|c|c|c|c|c|c|c|}
\hline \multirow[t]{3}{*}{ Oils } & \multirow{3}{*}{$\begin{array}{c}\text { Dose } \\
(\mathrm{ml} \\
\mathrm{kg}^{-1} \\
\text { seed) }\end{array}$} & \multicolumn{16}{|c|}{ Storage period ( Months) } \\
\hline & & \multicolumn{4}{|c|}{$\mathbf{0}$} & \multicolumn{4}{|c|}{2} & \multicolumn{4}{|c|}{4} & \multicolumn{4}{|c|}{6} \\
\hline & & \multicolumn{2}{|c|}{$\begin{array}{c}\text { Incidence } \\
(\%)\end{array}$} & \multicolumn{2}{|c|}{$\begin{array}{l}\text { Germination } \\
\qquad \%)\end{array}$} & \multicolumn{2}{|c|}{$\begin{array}{l}\text { Incidence } \\
\qquad(\%)\end{array}$} & \multicolumn{2}{|c|}{$\begin{array}{c}\text { Germination } \\
(\%)\end{array}$} & \multicolumn{2}{|c|}{$\begin{array}{c}\text { Incidence } \\
(\%)\end{array}$} & \multicolumn{2}{|c|}{$\begin{array}{l}\text { Germination } \\
\qquad \%)\end{array}$} & \multicolumn{2}{|c|}{$\begin{array}{c}\text { Incidence } \\
\qquad \%)\end{array}$} & \multicolumn{2}{|c|}{$\begin{array}{c}\text { Germination } \\
(\%)\end{array}$} \\
\hline Neem oil & 10.0 & 5.0 & (12.92) & 86.0 & $(68.15)$ & 5.75 & $(13.86)$ & 86.0 & $(68.06)$ & 4.0 & $(11.54)$ & 84.75 & $(67.05)$ & 2.5 & $(9.10)$ & 87.25 & $(69.16)$ \\
\hline $\begin{array}{c}\text { Mustard } \\
\text { oil }\end{array}$ & 10.0 & 6.5 & $(14.76)$ & 79.0 & $(62.74)$ & 6.00 & $(14.18)$ & 83.0 & $(65.71)$ & 5.0 & $(12.92)$ & 82.00 & $(64.95)$ & 3.5 & $(10.76)$ & 85.00 & $(67.24)$ \\
\hline Castor oil & 10.0 & 7.5 & (15.89) & 79.0 & $(62.76)$ & 8.00 & $(16.43)$ & 79.0 & $(62.77)$ & 6.0 & (14.18) & 80.00 & $(63.45)$ & 4.5 & (12.23) & 77.50 & $(61.74)$ \\
\hline Palas oil & 10.0 & 8.0 & (16.41) & 76.0 & $(60.68)$ & 8.50 & (16.94) & 78.0 & (62.04) & 6.5 & (14.76) & 78.50 & $(62.45)$ & 4.0 & (11.54) & 84.00 & $(66.45)$ \\
\hline Inoculated & & 10.0 & (18.43) & 72.0 & $(58.06)$ & 12.00 & $(20.27)$ & 75.0 & $(60.02)$ & 11.5 & (19.82) & 71.00 & $(57.43)$ & 9.0 & $(17.46)$ & 75.00 & $(60.03)$ \\
\hline $\begin{array}{c}\text { Un } \\
\text { Inoculated }\end{array}$ & & 7.0 & (15.34) & 78.0 & $(62.04)$ & 9.00 & (17.46) & 69.0 & $(56.17)$ & 6.0 & (14.13) & 80.50 & $(63.92)$ & 5.5 & (13.55) & 77.50 & $(61.75)$ \\
\hline SEm \pm & & & 0.26 & & 0.88 & & 0.18 & & 0.85 & & 0.33 & & 1.14 & & 0.29 & & 1.06 \\
\hline CD at $\mathbf{5 \%}$ & & & 0.77 & & 2.62 & & 0.52 & & 2.53 & & 0.99 & & 3.38 & & 0.85 & & 3.16 \\
\hline CV (\%) & & & 3.30 & & 2.73 & & 2.14 & & 2.73 & & 4.58 & & 3.60 & & 4.62 & & 3.30 \\
\hline
\end{tabular}

\begin{tabular}{|c|c|c|c|c|c|c|c|}
\hline \multicolumn{4}{|c|}{ Interaction of treatment and month for per cent incidence } & \multicolumn{4}{|c|}{ Interaction of treatment and month for per cent germination } \\
\hline SOV & $\mathrm{SEm} \pm$ & $\mathrm{CD}$ at $5 \%$ & $\mathrm{CV}(\%)$ & SOV & SEm \pm & $\begin{array}{c}\text { CD at } 5 \\
\%\end{array}$ & CV $(\%)$ \\
\hline $\mathbf{O}$ & 0.135 & 0.380 & 3.650 & $\mathrm{O}$ & 0.50 & 1.40 & 3.14 \\
\hline $\mathbf{M}$ & 0.102 & 0.288 & & M & 0.37 & 1.06 & \\
\hline OXM & 0.270 & 0.761 & & OXM & 0.99 & 2.79 & \\
\hline
\end{tabular}


With regard to interaction effect between six oils and four storage periods it was observed that minimum incidence of $F$. semitectum was in neem oil treated seed $(9.10 \%)$ following by mustard oil $(10.76 \%)$ at 6 months of storage. Maximum per cent seed germination was recorded in neem oil at six month of storage $(69.16 \%)$ followed by mustard oil and castor oil at 6 month storage respectively. These treatments were highly significant to each other as compared to inoculated untreated control (Table 4).

Traditional seed dressing material used in present study were tulsi leaf powder, mehandi leaves powder, turmeric powder and leaf powder of neem plant. Out of these neem leaves powder followed by tulsi leaf powder gave effective control of the seed mycoflora (A. alternata and $F$. semitectum) even after 6 month of storage and increased the seed germination. The effectiveness of these may be due to the presence of antifungal constituent in the form of phenolic substance and resinous gums and non volatile substance of unknown nature as observed by Skinner (1995) and Sharma (2001) who also noticed that pelleting of seed with $D$. stramonium, $A$. indica and $M$. exotica leaf extracts gave control of seed borne pathogenic fungi ( $A$. niger, A. alternata, $R$. solani, $M$. phaseolina and Fusarium oxysporum $\mathrm{f} \mathrm{sp}$. pisi) of pea as well as increased seed germination, seedling vigour and reducing seedling mortality. Meena and Mariappan (1994) also revealed that dry neem leaf powder as effective against seed borne fungi of sorghum. Jaimain (2003) found it effective in controlling of $M$. phaseolina in clusterbean.

In the present investigation, all the oils under test were found significantly better over the control as they minimized the mean per cent incidence of $A$. alternata and $F$. semitectum at each storage period. Incidence of $A$. alternata and $F$. semitectum was observed to be less in seeds treated with neem oil followed by mustard oil. Percentage of seed germination was also observed to be highest in seeds treated with neem and mustard oils. Use of oils as seed treatment has been found to be effective in avoiding the fungal invasion in pearl millet and also in cumin by Jain et al., (1998) and Anonymous (2001), respectively.

Therefore, further studies are warranted to deploy these usefully as seed/grain treatment to avoid the occurrence of mycoflora contamination. Present study, has shown that neem leaf powder and oil prevent or practically reduce the incidence of mycoflora like $A$. alternata and $F$. semitectum

\section{Acknowledgement}

Authors are thankful to the Agharkar Research Institute, Pune, Maharashtra for confirmation of identifications of the mycoflora isolated during course of investigation and to the Department of Plant Pathology, SKN College of Agriculture, Jobner (SK Rajasthan Agricultural University, Bikaner) for providing funds and facilities.

\section{References}

Anonymous, 2001. Controlling Bacterial and Fungal Diseases in Crops: Farmers Wisdom (Part II), Synthesis, Honey Bee 2 and $3: 3-4$ and 10

Anonymous, 2007-18.3 Directorate of Horticulture, Area and Production, State wise level area and production 2017-18.

Bajaya Tejpal, R.R. Ahir, R.P. Ghasolia, Mamta Bajya and Meena Choudhary (2017). Status of Alternaria Leaf spot of Blond psyllium (Plantago ovata) and its management through fungicides. Journal of Pharmacognosy and Phyto Chemistery. 6(4): 602-604. 
Choudhary Sajjan, 2015. Incidence and management of Alternaria sp. associated with Blond psyllium (Plantago ovate L.) seeds. M.Sc.(Ag.) Plant Pathology Thesis, SKNAU, Jobner.

Chourasia, H.K.1995. Mycobiota and mycotoxins in herbal drugs of Indian Pharmaceutical industries. Mycol. Res. 99 (6):697-703

Elwakil, M.A. and Ghoneem, K.M. 1999. Detection and Location of seed borne fungi 06 Blonde Psyllium and their transmission in seedlings. Pakistan journal of Biological Science (Pakistan).Vol. 2 (1) P.38-44.

Jaiman, R. K. 2003. Incidance of root rot causing fungi clusterbean [Cyamopsis tetragonoloba (L.) Taub] seeds their pathogenicity and management Ph.D thesis, Rajasthan Agricultural University, Bikaner Campus: Jobner

Jain, S.C.; Pathak, V.N. and Jain, K.L. 1998. Effect of some edible and non-edible oils on fungal invasion of pearlmillet seeds and their germination. J. Mycol. Pl. Pathol. 28: 317-318.

Meena, S.S. and Mariappan, V. 1994. Effect of plant leaf powders and containers on germination of mycoflora infested sorghum seeds. Chpater - 44 in crop diseases - Innovative Techniques and Management. Edited by K. Sivaprakasham and K. Seetharaman. Kalyani Publishers, New Delhi. 331333 pp.

Sharma, P.K. 2001. Micro-organisms associated with seed of pea (Pisum sativum L.) their pathogenic potential and disease management. Ph.D. (Ag.) Thesis, MPUA\&T, Udaipur.

Sinha, K.K. and Punam Kumari 1990. Some physiological abnormalities induced by Afl.B 1 in moong seeds (Vigna radiata var. Pusa Baishkhi). Mycopathologia. 110:77-79.

Skinner, F.R. 1995. In : K peach and M.V. tracey (eds.) Modern methods of plant analysis Vol. III. Sporinger verlay viertew Band pp. 626-725.

\section{How to cite this article:}

Meena, M. L., R. P. Maharshi and Bairwa, S. K. 2019. Efficacy of Traditional Seed Dressers and Oils on Isabgol Seeds against Alternaria alternata and Fusarium semitectum under Storage Conditions. Int.J.Curr.Microbiol.App.Sci. 8(10): 1467-1475.

doi: https://doi.org/10.20546/ijcmas.2019.810.172 